\title{
Changes in the air cell volume of artificially incubated ostrich eggs
}

\author{
Z. Brand $^{1,2 \#}$, S.W.P. Cloete ${ }^{1,3}$, I.A. Malecki ${ }^{1,4,5}$ \& C.R. Brown ${ }^{6}$ \\ ${ }^{1}$ Department of Animal Sciences, University of Stellenbosch, Private Bag X1, Matieland, 7602, South Africa \\ ${ }^{2}$ Institute for Animal Production, PO Box 351, Oudtshoorn, 6220, South Africa \\ ${ }^{3}$ Institute for Animal Production, Private Bag X1, Elsenburg, 7607, South Africa \\ ${ }^{4}$ School of Animal Biology, Faculty of Natural and Agricultural Science, University of Western Australia, Crawley, \\ 6009, Australia \\ ${ }^{5}$ UWA Institute of Agriculture, The University of Western Australia, 35, Stirling Highway, Crawley, WA 6009, \\ Australia \\ ${ }^{6}$ Institute of Science \& the Environment, University of Worcester, Henwick Grove, Worcester, WR2 6AJ, England
}

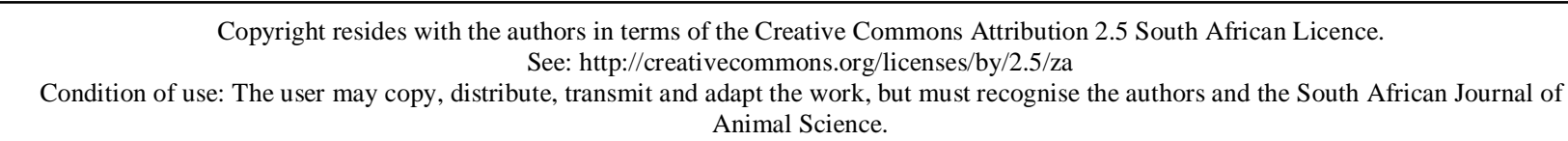

\begin{abstract}
A total of 2160 images of candled, incubated ostrich eggs were digitized to determine the percentage of egg volume occupied by the air cell at different stages of incubation. The air cell on average occupied $2.5 \%$ of the volume of fresh eggs. For eggs that hatched successfully, this volume increased to an average of $24.4 \%$ at 41 days of incubation, just prior to hatching. Air cell volume at 29 days of incubation for infertile eggs (19.3\%) was significantly higher when compared to dead-in-shell (DIS) eggs (14.3\%) and eggs that hatched (13.8\%). There was a significantly larger air cell volume in eggs that hatched normally compared with DIS eggs at 41 days of incubation (28.3\% vs. $21.7 \%$, respectively). No differences in air cell volume were observed up to day 17 of incubation for eggs that hatched normally between eggs that exhibited high, average or low rates of water loss, but from 20 days of incubation the air cell volume was significantly larger for high weight loss eggs. However, for the DIS eggs, air cell volume was consistently larger in eggs that exhibited high rates of water loss. Air cell volume was largely independent of adult strain (SA Black or Zimbabwean Blue) or whether chicks were assisted to hatch. Although some subtle differences in air cell size were detected between hatched and DIS chicks during this study, it is unlikely to find useful application in the broader industry.
\end{abstract}

Keywords: Water loss, artificial incubation, fertility, dead-in-shell, hatch, genotype

\# Corresponding author: zanellb@elsenburg.com

\section{Introduction}

Ostrich farming is a major agricultural enterprise in South Africa. The usual commercial practice is to keep breeding birds in large flocks or as pairs in small enclosures. To maximize production, eggs are collected daily, and incubated artificially. With hatching rates of only 50\% - 60\% (Brown et al., 1996; Deeming \& Ar, 1999; Van Schalkwyk, 2000), the success rate of artificially incubated ostrich eggs is low when compared with the hatching success of commercially reared chickens (90\% - 95\%), turkeys (75\% 77\%) and ducks (65\% - 82\%) (Hodgetts, 1990; Deeming, 1999). The low hatching success represents a considerable loss of production, and is a cause of concern in the local industry. Poor understanding of the pattern of embryonic development in ostriches may contribute to the poor hatching results reported previously.

An important tool for identifying incubation problems that cause low hatchability is knowledge of the age and degree of development of the embryo at the time of death (Ar \& Gefen, 1998). On commercial 
ostrich farms, egg candling is commonly used during artificial incubation to determine fertility and monitor the progress of the developing embryo. Candling, however, is not considered an effective tool to assess embryonic development or potentially to identify developmental problems at an early stage of incubation. Bradley (1998) found detection of infertile eggs by candling to be reasonably reliable on day 14, but it became increasingly difficult to distinguish between live and dead embryos as incubation progressed. Deeming (1995) reported only an increase in dark shadows as incubation progressed using the candling method.

One feature, however, that is usually easily distinguishable is the air cell at the blunt end of the egg. The air cell is initially formed between the two shell membranes as the egg cools after being laid. Air cell volume consequently increases in size during incubation as water is lost from the egg. The rate of water loss, and thus change in air cell volume, can potentially be influenced by any deviation from normal embryonic development. It is thus hypothesized that measurement of the air cell volume on specific days of the artificial incubation process may provide farmers with a simple tool to determine whether embryonic development is proceeding normally, as well as to estimate the age of the developing embryo or the age at which embryonic mortality occurs. This study aimed to determine the potential relationship between the development of ostrich embryos and air cell volume at various stages of the artificial incubation process, as well as report on factors influencing air cell volume during the incubation period.

\section{Material and Methods}

Data obtained for this study were recorded during the 2007 breeding season for eggs that originated from the commercial ostrich breeding flock at the Oudtshoorn Research Farm, outside Oudtshoorn in South Africa. The origin of birds, their husbandry and artificial incubation practices for the eggs have been described by Van Schalkwyk et al. (1996), Van Schalkwyk (1998) and Bunter \& Cloete (2004). All eggs collected during the 2007 breeding season were weighed and identified by date and paddock (female) of origin. Methods for collection, disinfection and storage at the experimental site are well documented (Van Schalkwyk et al., 1998; 1999; Brand et al., 2007). Data involved various combinations of the two purebred bloodlines South African Blacks (SAB) and Zimbabwean Blues (ZB), as well as ZB male x SAB female crosses.

It is common practice in the South African ostrich industry to candle eggs, usually on day 21 of the artificial incubation period to establish whether they are fertile, whereas the transition from the incubator to the hatcher usually takes place on day 35 of incubation.

One hundred and twenty fresh eggs (chosen at random to represent eggs at that stage) were weighed and candled. Candling was performed with a 100 Watt candling light that was placed directly under the egg to illuminate its internal content. Candling enabled visualization of the internal detail of the egg through the eggshell. During the process of candling, eggs were photographed with a Pentax P30T $35 \mathrm{~mm}$ single lens reflex camera equipped with a 35 - $80 \mathrm{~mm}$ zoom lens mounted on a tripod. Images were captured at a shutter speed of one second at an aperture of $\mathrm{f} 4$ on ISO 100 film. These eggs were set and incubated in an electronic Buckeye ${ }^{\circledR}$ incubator at a temperature of $36{ }^{\circ} \mathrm{C}$ and relative humidity of $24 \%$ to investigate the changes in air cell volume and the development of the embryo. Eggs were candled and photographed every 2 - 3 days throughout the 42-day incubation period to monitor air cell size. A total of 2160 images were digitized from the photographs and evaluated by using the software package AnalySIS ${ }^{\circledR}$ (Soft Imaging System, 1999). The percentage of egg volume occupied by the air cell was determined, and the area occupied by the air cell (in pixels) was expressed as a percentage of the area occupied by the entire egg. Examples of images acquired in this way for embryos of 3 to 42 days of age are depicted in Figures 1a to $\mathrm{f}$. In practice, infertile eggs would be removed during candling at 21 days of incubation. In the present study, however, infertile eggs were not removed from the incubators, but retained for the entire 42-day incubation period to investigate the dynamics of change in the air cell volume of infertile eggs. Eggs were weighed on day 21 and on day 35 of incubation to determine weight loss of the eggs at that stage. Weight loss was calculated as the difference between the fresh egg weight and the egg weight recorded on days 21 and 35, respectively. On day 44 of incubation, fertilized eggs that did not hatch were candled to see if any movement of the chick could be detected, thus indicating whether internal pipping had occurred. All the eggs that did not hatch were then manually opened at the air cell area, and the position of the embryo and point of internal pipping (if observed) noted. The rest of the eggs that failed to hatch were opened and the cause of mortality recorded. This included infertile eggs, as well as eggs with early and late embryo mortalities. 
The data were subjected to analysis using ASREML software (Gilmour et al., 1999). The software is suitable for fitting a wide range of fixed and random effects, while least-square means for selected systematic effects are predicted simultaneously. Fixed effects were tested for significance, using an F-test in the analysis of variance table. Fixed effects that were considered included the designation of the egg (hatched, infertile or dead-in-shell), as well as bloodline (SAB, ZB or ZB male x SAB female crosses). These effects were interacted with the appropriate number of days of incubation. Traits that were considered were percentage water loss up to 21 days of incubation (WL21), percentage water loss up to 35 days of incubation (WL35) and the percentage of egg occupied by the air cell. The random effect of egg was included in the analysis to account for the variation attributed to the repeated sampling of specific eggs.
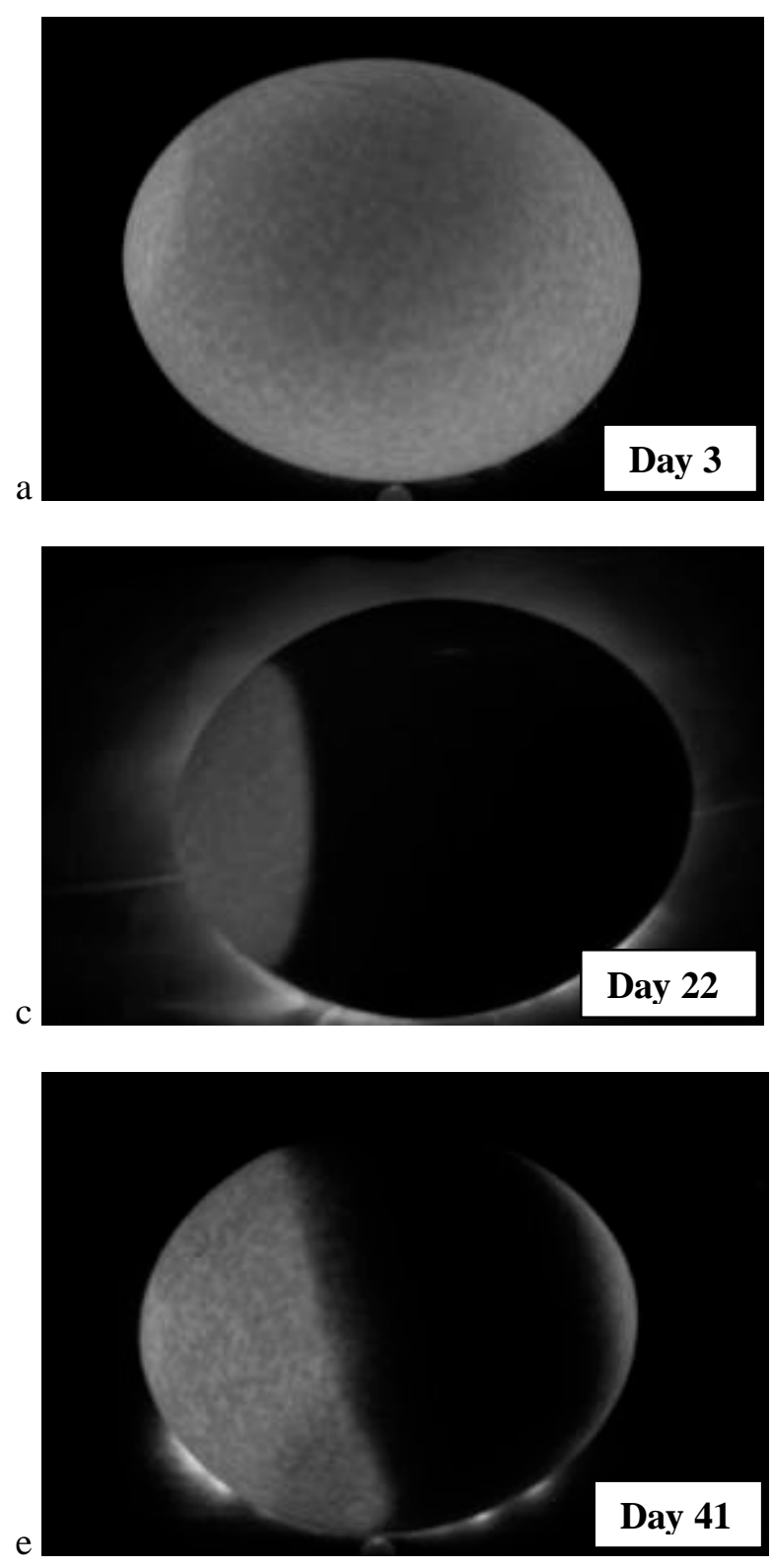
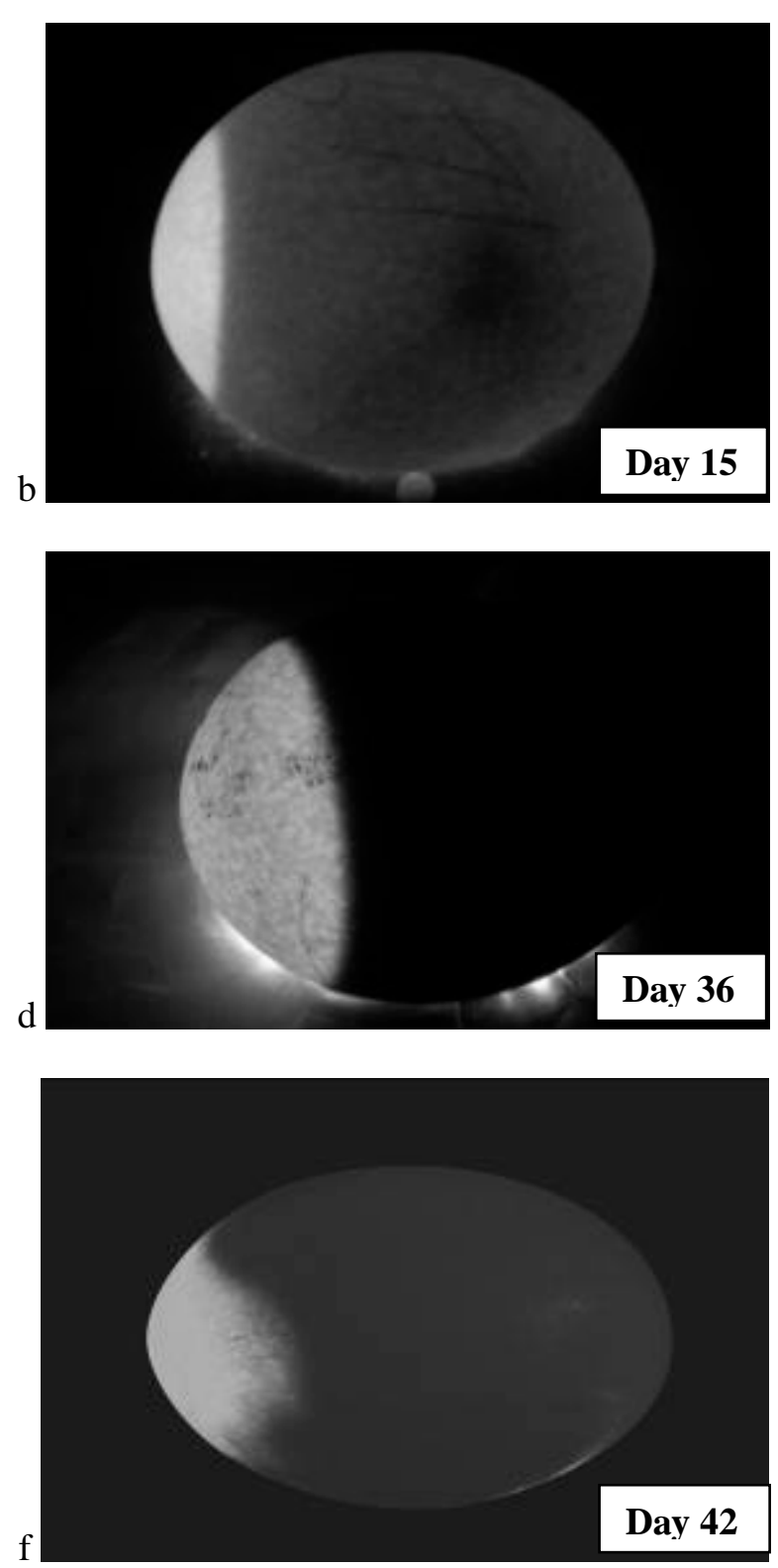

Figure 1 Illumination of ostrich eggs at specific stages (as indicated by photo legends a to f) of an artificial incubation period of 42 days. The lighter areas represent the air cell volume, and the dark area the developing ostrich embryo. 


\section{Results and Discussion}

The average weight of the 120 eggs used in this study was $1389 \pm 112 \mathrm{~g}$, with a coefficient of variation (CV) of $8.11 \%$ (Table 1). The mean was slightly lower than previous weights reported by Cloete et al. (2004), Bunter \& Cloete (2004) and Brand et al. (2008a; b) for eggs collected from the same resource flock. This observation could probably be attributed to the small sample of eggs used in the present study. Water loss ranged from $4.6 \%$ to $13.0 \%$ of fresh egg weight at day 21 of incubation, and $7.4 \%$ to $20.0 \%$ at day 35 of incubation, with higher CVs of $23.9 \%$ and $24.1 \%$, respectively. These results are in agreement with previous results reported by Brand et al. (2008a; b) for eggs collected from the same resource flock. The percentage of the egg occupied by the air cell varied considerably between eggs candled throughout incubation. Air cell volume averaged 10.9\% for eggs incubated for 21 days, and 18.5\% for eggs incubated for 35 days with high corresponding CVs of $31.5 \%$ and 31.9\%, respectively. No comparable estimates could be found in the literature.

Table 1 Descriptive statistics for traits recorded from eggs of breeding ostrich females for the 2007 production year

\begin{tabular}{lrcc}
\hline Traits & Mean \pm SD & CV (\%) & Range \\
\hline EWT (g) & $1389 \pm 112$ & 8.1 & $1121-1765$ \\
WL21 (\% of fresh egg weight) & $7.5 \pm 1.8$ & 23.9 & $4.6-12.6$ \\
AC 21(\%) & $10.9 \pm 3.4$ & 31.5 & $4.5-20.0$ \\
WL35 (\% of fresh egg weight) & $12.4 \pm 3.0$ & 24.1 & $7.4-20.2$ \\
AC 35(\%) & $18.5 \pm 5.9$ & 31.9 & $9.2-39.7$
\end{tabular}

SD: standard deviation; CV\%: coefficient of variation; EWT: egg weight at time of lay; WL21: water loss at day 21 of incubation; AC 21\%: air cell as \% of egg volume at 21 days of incubation; WL35: water loss at day 35 of incubation; AC 35\%: air cell as \% of egg volume at 35 days of incubation.

The proportion of the egg occupied by the air cell increased throughout the incubation period of 42 days (Figure 2). The average percentage of the egg occupied by the air cell for fertile eggs that hatched successfully was $2.5 \%$ for fresh eggs. This increased steadily to $24.4 \%$ at day 42 of incubation. By day 28 of incubation, the dark shadowed area of the developing embryo covered almost the entire eggshell, with the exception of the air cell region in fertile eggs. From day 29 of incubation and longer, air cell volume for infertile eggs was significantly higher $(19.3 \%, P<0.05)$ when compared with that observed for dead-in-shell (DIS) eggs (14.3\%) and eggs that hatched successfully (13.8\%). There appeared to be a plateau in the expansion of the air cell area from day 17 to day 27 of incubation. This trend was similar to findings reported by Deeming et al. (1993). The occurrence of this plateau cannot be elucidated, for the embryo increased substantially in both weight and length during this period (Deeming \& Ar, 1999).

A rapid increase in air cell volume towards the end of incubation was similarly observed by Jarvis et al. (1985) and Deeming (1995) when measuring the air cell volume. This increase in air cell volume from day 38 of incubation could be attributed to an increase in embryonic heat production, which in turn leads to an increase in egg temperature, contributing to increased water loss. The most pronounced increase in $\mathrm{O}_{2}$ consumption of ostrich eggs occurred between 26 and 31 days of incubation, as reported by Van Schalkwyk et al. (2002). This, together with a similar increase in embryonic metabolism at day 25 of incubation (Reiner \& Dzapo, 1994), could contribute to the increase in air cell volume towards the end of incubation. The first indication of the commencement of hatching took place on about 42 days of incubation with the appearance of a shadow on one side of the air cell as the chick pipped internally (Figure 1f). Movement of the chick could be observed throughout internal pipping until external pipping occurred when the chick broke through the egg shell.

Air cell volume was generally independent of genotype, with eggs from the SAB, ZB and ZB x SAB genotypes generally showing the same basic pattern. Occasional significant $(P<0.05)$ differences between genotypes could be ascribed to sampling, as a small number of eggs were included for the ZB strain in 
particular. Air cell volumes of those eggs that hatched naturally and those with chicks that were assisted to hatch were accordingly similar, without any significant $(P<0.05)$ differences throughout the period of incubation.

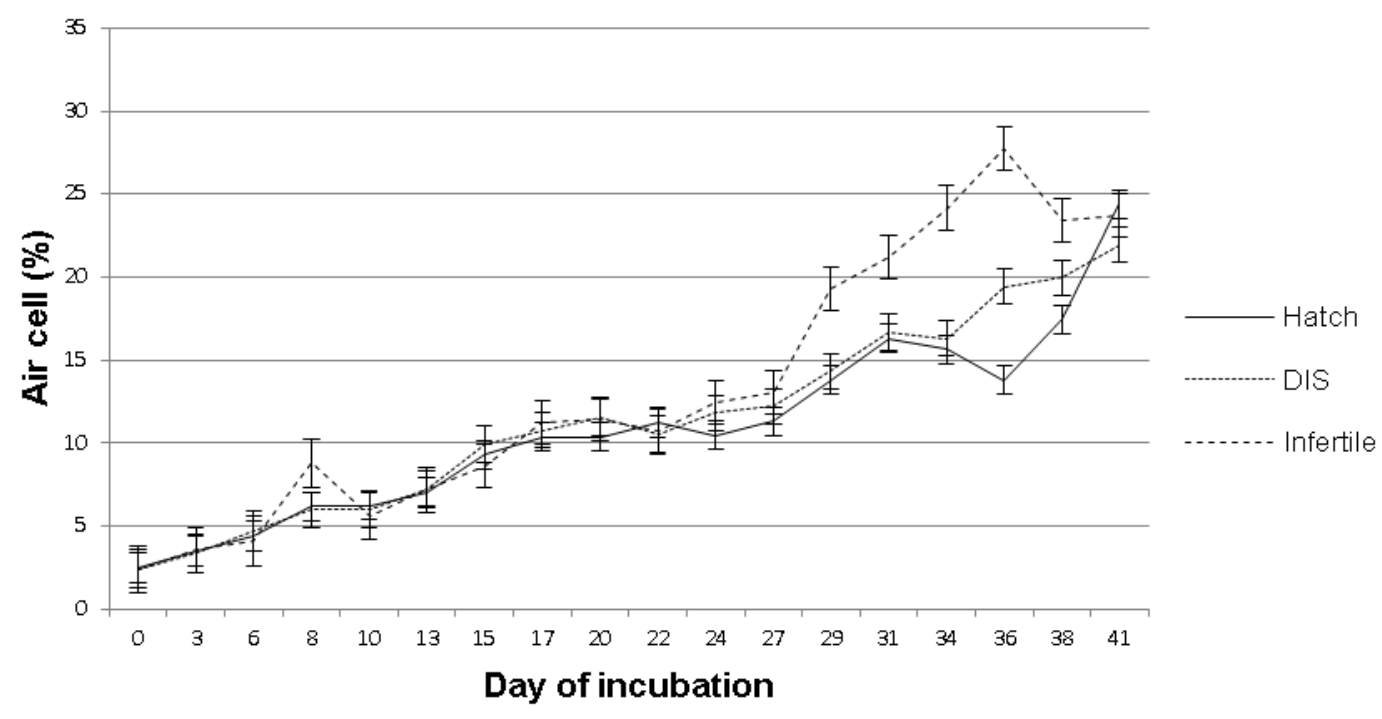

Figure 2 Percentage of egg volume ( \pm S.E.) occupied by the air cell in hatched, infertile and dead-in-shell ostrich eggs during the 42-day incubation period.

Air cell volumes of DIS eggs with a low WL (4.37\% - 14.8\%) followed the same trend as that of hatched eggs $(3.36 \%-16.3 \%)$ from the initiation of the incubation process up to 38 days of incubation (Figure 3a). A pronounced increase in air cell volume $(P<0.05)$ occurred at 41 days of incubation for eggs that hatched normally (21.9\% and $24.4 \%$, respectively, for DIS and hatched eggs). This increase in air cell volume could potentially be attributed to an increase in water loss following internal pipping and the consequent rupture of the shell membranes. No differences in air cell volume were found between DIS and hatched eggs for the medium WL $(2.49 \%-22.3 \%$ and $2.6 \%-23.0 \%$, respectively) group for the entire incubation period, the only exception being for 38 days of incubation (Figure 3b). Air cell volume for the high WL group (1.41\% - 22.6\%) was generally higher throughout for the DIS eggs, especially on days 15, 24 and 38 of incubation $(P<0.05)$. At 41 days of incubation, the air cell volume of the hatched eggs increased relative to the air cell volume observed for DIS eggs (22.6\% and 26.9\%; Figure 3c).

As stated, increased WL occurred with the initiation of internal pipping, which was also observed for those dead-in-shell eggs where chicks pipped internally. There were no significant differences between the air cell volume of eggs that experienced high, average and low levels of WL up to day 20 of incubation. From day 20 to day 38 of incubation the high WL group also had a larger air cell volume (16.2\% - 24.2\%), whereas from 21 days of incubation the air cell volume for the eggs with low WL was significantly smaller if compared with the average and high WL eggs. Air cell volumes for hatched eggs for the low WL group were $14 \%-16 \%$ for days 34 and 36 of incubation, respectively, and did not differ from those reported for the average WL group (15.5\% - 17\%; $P>0.05)$. These results were consistent with findings published by Swart \& Rahn (1988), Blood et al. (1998) and Brand et al. (2008a; b), where a water loss of $13 \%$ in ostrich eggs up to 35 days of incubation was reported.

At $16.8 \%-22 \%$ for days 34 and 36 of incubation, respectively, air cell volumes for the high WL group were larger $(P<0.05)$ compared with the lower and medium WL groups. From day 38 of incubation, the air cell volume of the low WL group that hatched increased rapidly and, together with the high WL group, was significantly higher at 41 days of incubation than the average WL group. When compared with the low and high water loss groups in eggs that hatched, water loss for all the DIS eggs was $~ 2 \%$ lower and $\sim 3 \%$ higher. Deeming (1995) was not able to establish a significant difference in air cell volume between hatched and DIS eggs. The air cell volume of the low WL DIS group increased rapidly in size at day 39 of 
incubation, while no significant difference was found between the air cell volumes of the various levels of WL at 41 days of incubation. The air cell volume of DIS eggs candled at day 43 to 44 of incubation remained round in appearance, with no movement of the chick detected.

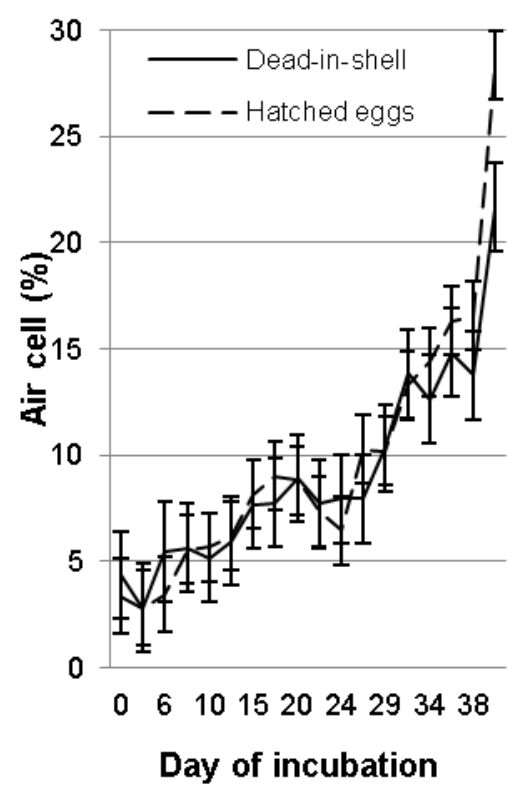

Figure 3 Percentage of egg volume occupied by the air cell in dead-in-shell and hatched ostrich eggs during the 42-day incubation period for eggs with low a), medium b), and high c) water loss.

\section{Conclusions}

Egg candling is a useful tool to assess embryo development, and to identify infertile eggs, as well as early embryonic mortalities in ostrich embryos. In this study, the aim was to determine the potential of the technique to assist with determining embryonic mortality at later stages. However, while changes in the air cell volume conformed to expectations, differences between hatched eggs and those with dead-in-shell chicks were generally not significant. Even when significant, such differences were unlikely to be observed outside a rigorous experiment, such as this. Fairly large levels of variation observed within categories involving hatched and dead-in-shell chicks, as well as infertile eggs, complicating the formulation of robust guidelines that could be used in commercial hatcheries to identify embryonic mortalities where it is unlikely that such detailed parameters will be recorded. It can thus be concluded that, although this study was able to detect subtle differences between hatched and DIS chicks, the use of air cell volume to predict embryo development throughout the hatching period is unlikely to find application in the broader industry.

\section{Acknowledgements}

We acknowledge the financial and infrastructure support of the Western Cape Department of Agriculture, as well as the financial support of the Western Cape Agricultural Research Trust. The authors also thank Isak Janse for the maintenance of the incubators and for the eggs, as well as everyone who provided assistance throughout the project.

\section{References}

Ar, A. \& Gefen, E., 1998. Further improving hatchability in artificial incubation of ostrich eggs. Proc. $2^{\text {nd }}$ Int. Ratite Conf., 21-25 September 1998, Oudtshoorn, South Africa. pp. 141-147.

Blood, J.R., Van Schalkwyk, S.J., Cloete, S.W.P. \& Brand, Z., 1998. Embryonic deaths in relation to water loss of artificially incubated ostrich eggs. Proc. $2^{\text {nd }}$ Int. Ratite Conf. Oudtshoorn, South Africa. pp. 148-151. 
Bradley, A., 1998. Boosting ostrich production through better egg hatchability. Rural Industries Research and Development Corporation. RIRDC Publication no. 98/17.

Brand, Z., Cloete, S.W.P., Brown, C.R. \& Malecki, I.A., 2007. Factors related to shell deaths during artificial incubation of ostrich eggs. J. S. Afr. Vet. Assoc. 78, 195-200.

Brand, Z., Cloete, S.W.P., Malecki, I.A. \& Brown, C.R., 2008a. The genetic relationships between water loss and shell-deaths in ostrich eggs, assessed as traits of the dam. Aust. J. Exp. Agric. 48, 1326-1331.

Brand, Z., Cloete, S.W.P., Brown, C.R. \& Malecki, I.A., 2008b. Systematic factors that affect ostrich egg incubation traits. S. Afr. J. Anim. Sci. 38, 315-325.

Brown, C.R., Peinke, D. \& Loveridge, A., 1996. Mortality in near-term ostrich embryos during artificial incubation. Br. Poult. Sci. 37, 73-85.

Bunter, K.L. \& Cloete, S.W.P., 2004. Genetic parameters for egg-, chick- and live-weight traits recorded in farmed ostriches (Struthio camelus). Livest. Prod. Sci. 91, 9-22.

Cloete, S.W.P., Bunter, K.L., Brand, Z. \& Lambrechts, H., 2004. (Co)variances for reproduction, egg weight and chick weight in ostriches. S. Afr. J. Anim. Sci. 34, 17-19.

Deeming, D.C., 1995. The hatching sequence of ostrich (Struthio camelus) embryos with notes on development as observed by candling. Br. Poult. Sci. 36, 67-78.

Deeming, D.C., Ayres, L. \& Ayres, F.J., 1993. Observations on the commercial production of ostrich (Struthio camelus) in the United Kingdom: incubation. Vet. Rec. 132, 602-307.

Deeming, D.C., 1999. Factors affecting the success of commercial incubation. In: The Ostrich Biology, Production and Health. Ed. Deeming, D.C. CABI Publishing, Wallingford, Oxon, United Kingdom. pp. 159-190.

Deeming, D.C. \& Ar, A., 1999. Factors affecting the success of commercial incubation. In: The Ostrich: Biology, Production and Health. Ed: Deeming, D.C. CABI Publishing, Wallingford, Oxon, United Kingdom. pp. 275-292.

Deeming, D.C., Dick, A.C.K. \& Ayres, L.L, 1996. Ostrich Chick rearing. A Stockman's Guide. Ratite Conference, Oxfordshire, ISBN 0-952758-1-5. pp. 5-14.

Gefen, E. \& Ar, A., 2001. Morphological description of the developing ostrich embryo: a tool for embryonic age estimation. Israel J. Zool. 47, 87-97.

Gilmour, A.R., Cullis, B.R., Welham, S.J. \& Thompson, R., 1999. ASREML - Reference manual. NSW Agriculture Biometric Bulletin No. 3 NSW Agriculture, Orange agriculture Institute, Forest Road, Orange 2800, NSW, Australia.

Hodgetts, B., 1990. Current hatchabilities in species of domestic importance and the scope for improvement. In: Avian Incubation. Ed. Tullett, S.G., CAB Direct. pp. 139-144.

Jarvis, M.J.F., Keffen, R.H. \& Jarvis, C., 1985. Some physical requirements for ostrich egg incubation. Ostrich 56, 42-51.

Reiner, G. \& Dzapo, V., 1994. The oxygen consumption of ostrich embryos during incubation and its significance for chick vitality. Ostrich News, December 1994. pp. 49-58.

Soft Imaging System, 1999. AnalySIS ${ }^{\circledR}$ User's guide. Soft Imaging System GmbH, Hammer Street 89, D48153 Münster, Germany.

Swart, D. \& Rahn, H., 1988. Microclimate of ostrich nests: Measurements of egg temperature and nest humidity using egg hygrometers. J. Comp. Physiol. B. 157, 845-853.

Van Schalkwyk, S.J., 1998. Improvement of fertility and hatchability of artificially incubated ostrich eggs in the Little Karoo. MSc thesis, Rhodes University, Grahamstown, South Africa.

Van Schalkwyk, S.J., Brand, Z., Cloete, S.W.P. \& Brown, C.R., 1999. Effects of time of egg collection and pre-incubation treatment on blastoderm development and embryonic mortality in ostrich embryos. S. Afr. J. Anim. Sci. 29, 154-163.

Van Schalkwyk, S.J., Cloete, S.W.P., Brown, C.R. \& Brand, Z., 2000. Hatching success of ostrich eggs in relation to setting, turning and angle of rotation. Br. Poult. Sci. 41, 46-52.

Van Schalkwyk, S.J., Cloete, S.W.P. \& Brown, C.R., 2002. Gas exchange of the ostrich embryo during peak metabolism in relation to incubator design. S. Afr. J. Anim. Sci. 32, 122-129. 